\title{
A Combinatorial Model for q-Generalized Stirling and Bell Numbers
}

\author{
Miguel Méndez ${ }^{1}$ and Adolfo Rodríguez ${ }^{2}$ \\ ${ }^{1}$ Departamento de Matemática, Instituto Venezolano de Investigaciones Científicas, A.P. 21827, Caracas 1020-A, \\ Venezuela. \\ ${ }^{2}$ Laboratoire de Combinatoire et d'Informatique Mathématique (LaCIM), Université du Québec à Montréal, CP \\ 8888, Succ. Centre-ville Montréal (Québec) H3C 3P8, Canada.
}

\begin{abstract}
We describe a combinatorial model for the $q$-analogs of the generalized Stirling numbers in terms of bugs and colonies. Using both algebraic and combinatorial methods, we derive explicit formulas, recursions and generating functions for these $q$-analogs. We give a weight preserving bijective correspondence between our combinatorial model and rook placements on Ferrer boards. We outline a direct application of our theory to the theory of dual graded graphs developed by Fomin. Lastly we define a natural $p, q$-analog of these generalized Stirling numbers.
\end{abstract}

Keywords: Stirling, Bell, boson, $q$-analog, rook numbers, dual graded graphs

\section{Introduction}

Generalized Stirling numbers appear as the coefficients of the normal ordering of a word in the boson creation and annihilation operators $a^{\dagger}$ and $a$, satisfying the commutation rule $\left[a, a^{\dagger}\right]=1$. An interpretation of these coefficients as rook numbers was given by Navon [10]. In this paper we extend the combinatorial model developed in [9] in terms of bugs, colonies and settlements, by adding weights to these combinatorial structures. Based on this model, we obtain formulas for the $q$-generalized Stirling numbers, defined as the coefficients of the normal ordering of a word in the $q$-bosonic operators of type $M$ (see [6, 7, 8, 11, 12]) satisfying the commutation rule

$$
\left[a_{q}, a_{q}^{\dagger}\right]_{q}=a_{q} a_{q}^{\dagger}-q a_{q}^{\dagger} a_{q}=1
$$

In section 2 and 3 we use algebraic and combinatorial tools, respectively, to obtain explicit formulas, generating functions and recursive formulas for the $q$-generalized Stirling and Bell numbers. In section 4 we give a weight preserving bijection between colonies and rook placements, which relates our combinatorial model to the one described in [13]. In section 5 we show a direct application of the present theory to the theory of duality of graded graphs introduced by Fomin [14]. In section 6 we define $p, q$-analogs of the generalized Stirling numbers as a natural generalization of the $q$-analogs and derive a formula in terms of the $q$-analogs. We have also obtained a natural extension of this work to the context of combinatorial species. However, we will limit ourselves to elementary algebraic manipulations and basic combinatorial results, as the species approach would be too long to be discussed in this abstract. 


\section{$2 q$-analogs of the generalized Stirling numbers}

Given two sequences of positive integers $\mathbf{b}=\left(b_{1}, b_{2}, \ldots, b_{n}\right)$ and $\mathbf{f}=\left(f_{1}, f_{2}, \ldots, f_{n}\right)$, define $d_{j}=$ $\sum_{i=1}^{j}\left(b_{i}-f_{i}\right)$ for $j=1,2, \ldots, n$, and set $d_{0}=0$. Define the $q$-generalized Stirling numbers $S_{\mathbf{b}, \mathbf{f}}^{q}(k)$ as the coefficients (polynomials in $q$ ) that appear in the straightening formula:

$$
\left(a_{q}^{\dagger}\right)^{b_{n}} a_{q}^{f_{n}} \cdots\left(a_{q}^{\dagger}\right)^{b_{1}} a_{q}^{f_{1}}=\sum_{k=f_{1}}^{|\mathbf{f}|} S_{\mathbf{b}, \mathbf{f}}^{q}(k)\left(a_{q}^{\dagger}\right)^{d_{n}+k} a_{q}^{k} .
$$

where $|\mathbf{f}|=f_{1}+\cdots+f_{n}$. Note that expression (2) is normally ordered, and that the general problem of normal ordering of a $q$-boson string is reduced to computing the coefficients $S_{\mathbf{b}, \mathbf{f}}^{q}(k)$. The $q$-analog of the generalized exponential polynomials, sometimes also called Bell polynomials, are defined as

$$
\boldsymbol{\Phi}_{\mathbf{b}, \mathbf{f}}^{q}(x)=\sum_{k} S_{\mathbf{b}, \mathbf{f}}^{q}(k) x^{k}
$$

In turn, the $q$-generalized Bell numbers are defined as

$$
B_{\mathbf{b}, \mathbf{f}}^{q}=\sum_{k=f_{1}}^{|\mathbf{f}|} S_{\mathbf{b}, \mathbf{f}}^{q}(k)=\boldsymbol{\Phi}_{\mathbf{b}, \mathbf{f}}^{q}(1) .
$$

Recall that the $q$-analog of the derivative $D_{q}$ is given by:

$$
D_{q} f(x)=\frac{f(q x)-f(x)}{(q-1) x} .
$$

Now, denoting by $X$ the operator that multiplies a function by the variable $x$, we easily verify that $X$ and $D_{q}$ satisfy the relation

$$
D_{q} X=q X D_{q}+I .
$$

Thus, since $X$ and $D_{q}$ satisfy the same commuting relation as $a_{q}^{\dagger}$ and $a_{q}$, we get

$$
X^{b_{n}} D_{q}^{f_{n}} \ldots X^{b_{2}} D_{q}^{b_{2}} X^{b_{1}} D_{q}^{f_{1}}=\sum_{k} S_{\mathbf{b}, \mathbf{f}}^{q}(k) X^{d_{n}+k} D_{q}^{k}
$$

As usual, the $q$-analog $[m]$ of a natural number $m$ is $[m]=1+q+\cdots+q^{m-1}=\frac{1-q^{m}}{1-q}$, and the $q$ analog of the falling factorial $[m]_{k}$ is defined as $[m]_{k}=[m][m-1][m-2] \ldots[m-k+1]$. Clearly, $[m]_{m}=[m] !=[m][m-1] \ldots 1$. For integers $n \geq k \geq 0$, define the $q$-binomial coefficient

$$
\left[\begin{array}{c}
m \\
k
\end{array}\right]=\frac{[m] !}{[k] ![m-k] !}=\frac{[m]_{k}}{[k] !}
$$

We denote by $e_{q}^{x}$ and $E_{q}^{x}$, the well known $q$-analogs of the exponential function, given by

$$
e_{q}^{x}=\prod_{k=0}^{\infty} \frac{1}{1+(q-1) q^{k} x}
$$




$$
E_{q}^{x}=\prod_{k=0}^{\infty}\left(1-(q-1) q^{k} x\right)
$$

Equivalently $e_{q}^{x}=\sum_{m=0}^{\infty} \frac{x^{m}}{[m] !}$ and $E_{q}^{x}=\sum_{m=0}^{\infty} q^{\left(\begin{array}{c}m \\ 2\end{array}\right)} \frac{x^{m}}{[m] !}$. It is immediate from this that $\left(e_{q}^{x}\right)^{-1}=$ $E_{q}^{-x}$ and $D_{q} e_{q}^{x}=e_{q}^{x}$.

The result below follows from equation (7):

Proposition 1 Let $X$ and $D_{q}$ be operators acting on functions on a single variable $x$, defined by $X f(x)=$ $x f(x)$ and $D_{q} f(x)=\frac{f(q x)-f(x)}{(q-1) x}$. Let $\mathbf{b}=\left(b_{1}, \ldots, b_{n}\right), \mathbf{f}=\left(f_{1}, \ldots, f_{n}\right)$ be finite sequences of nonnegative integers. Set $d_{j}=\sum_{i=1}^{j}\left(b_{i}-f_{i}\right), d_{0}=0$ and $p_{\mathbf{b}, \mathbf{f}}^{q}(m)=\prod_{j=1}^{n}\left[m+d_{j-1}\right]_{f_{j}}$. We have

$$
\begin{aligned}
X^{b_{n}} D_{q}^{f_{n}} \cdots X^{b_{1}} D_{q}^{f_{1}} x^{m} & =p_{\mathbf{b}, \mathbf{f}}^{q}(m) x^{m+d_{n}} \\
p_{\mathbf{b}, \mathbf{f}}^{q}(m) & =\sum_{k} S_{\mathbf{b}, \mathbf{f}}^{q}(k)[m]_{k}
\end{aligned}
$$

where the $S_{\mathbf{b}, \mathbf{f}}^{q}(k)$ are the q-generalized Stirling numbers, uniquely determined by the equality

$$
X^{b_{n}} D_{q}^{f_{n}} \ldots X^{b_{2}} D_{q}^{b_{2}} X^{b_{1}} D_{q}^{f_{1}}=\sum_{k} S_{\mathbf{b}, \mathbf{f}}^{q}(k) X^{d_{n}+k} D_{q}^{k}
$$

This immediately implies the following statement

Corollary 1 (Dobinsky relations) Let $\boldsymbol{\Phi}_{\mathbf{b}, \mathbf{f}}^{q}(x)$ be the q-generalized exponential polynomial, given by

$$
\boldsymbol{\Phi}_{\mathbf{b}, \mathbf{f}}^{q}(x)=\sum_{k} S_{\mathbf{b}, \mathbf{f}}^{q}(k) x^{k}
$$

We have the identity

$$
\boldsymbol{\Phi}_{\mathbf{b}, \mathbf{f}}^{q}(x) e_{q}^{x}=\sum_{m} p_{\mathbf{b}, \mathbf{f}}^{q}(m) \frac{x^{m}}{[m] !} .
$$

We now obtain explicit formulas for the $q$-generalized Stirling and Bell numbers.

Corollary 2 (Explicit formulas) Let $B_{\mathbf{b}, \mathbf{f}}^{q}$ denote the q-generalized Bell numbers, defined by

$$
B_{\mathbf{b}, \mathbf{f}}^{q}=\boldsymbol{\Phi}_{\mathbf{b}, \mathbf{f}}^{q}(1)
$$

We have the formulas

$$
\begin{aligned}
S_{\mathbf{b}, \mathbf{f}}^{q}(k) & =\frac{1}{[k] !} \sum_{j=0}^{k}\left[\begin{array}{l}
k \\
j
\end{array}\right](-1)^{k-j} q^{\left(\frac{k-j}{2}\right)} p_{\mathbf{b}, \mathbf{f}}^{q}(j) \\
B_{\mathbf{b}, \mathbf{f}}^{q} & =\prod_{k=1}^{\infty}\left(1+(q-1) q^{k}\right) \sum_{m} \frac{p_{\mathbf{b}, \mathbf{f}}^{q}(m)}{[m] !}
\end{aligned}
$$




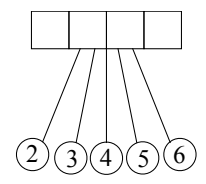

Fig. 1: Bug of type $(b, f)=(4,5)$.

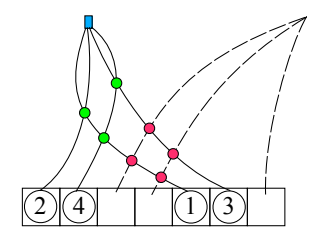

Fig. 2: Placement $p$ with $E(p)=4, I(p)=3$, and $w(p)=q^{7}$.

\section{The combinatorial approach}

Varvak points out in [13] that the general coefficient in the normal ordered form of a generic $q$-bosonic word can be interpreted as a $q$-rook number. A different approach is described in [8] in terms of $q$ weighted Feynman diagrams. In this section we introduce an alternative combinatorial model that gives a combinatorial interpretation of the $q$-generalized Stirling numbers in terms of colonies of bugs. Using this model we give a simple combinatorial proof of a recursive formula for the $q$-generalized Stirling numbers.

Definition $1 A$ bug of type $(b, f)$ consists of a body and $f$ legs (see figure 1). The body is formed by $b$ linearly ordered empty cells. The legs are arcs attached to the body, but not related to its cells. Each foot (bottom end) of the $f$ legs is labelled with one number of an integer segment $(m, m+f]=\{m+1, m+$ $2, \ldots, m+f\}$. A worm is a bug with no legs $(f=0)$ and a ghost is a bug with no body cells $(b=0)$.

Definition 2 Consider two totally ordered sets $\left(A, \leq_{A}\right)$ and $\left(B, \leq_{B}\right)$, where $|A| \leq|B|$. A placement $p$ of the elements of $A$ into the elements of $B$ is an injective function $p: A \rightarrow B$.

An internal crossing of $p$ is a pair of elements $a_{1}$ and $a_{2}$ in $A$, such that $a_{1}<_{A} a_{2}$ and $p\left(a_{1}\right)>_{B} p\left(a_{2}\right)$. An external crossing of $p$ is a pair $(a, b), a \in A$ and $b \in B$, such that $p^{-1}(b)=\emptyset$ and $p(a)>_{B} b$. A placement could be represented as one of the possible ways of placing each of the elements of the set $A$ of feet of a ghost bug, into the elements of the set $B$ of cells of a worm. The internal crossings are pictorially represented as the crossings between legs of the ghost. The external crossings as the crossings of the legs of this ghost with the legs of an additional ghost bug whose unlabelled feet are placed in the empty cells (see figure 2). The weight of a placement $p$ is defined to be

$$
w(p)=q^{I(p)+E(p)},
$$

where $I(p)$ and $E(p)$ respectively denote the number of internal and external crossings of $p$. For a set $P$ of placements, define the q-cardinality of $P$ as $|P|_{q}=\sum_{p \in P} w(p)$. 
Proposition 2 Denote by $P_{m, k}$ the set of placements (injective functions) of a partially ordered $k$-element set $A$ into a partially ordered $m$-element set $B$, and by $\widetilde{P}_{m, k}$ the subset of $P_{m, k}$ containing only placements with no internal crossings. We have

$$
\begin{aligned}
\left|P_{m, k}\right|_{q} & =[m]_{k} \\
\left|\widetilde{P}_{m, k}\right|_{q} & =\left[\begin{array}{c}
m \\
k
\end{array}\right] .
\end{aligned}
$$

Proof: To prove 177 observe that $\left|P_{m, 1}\right|_{q}=1+q+q^{2}+\cdots+q^{m-1}=[m]$. Therefore, all we have to prove is that $\left|P_{m, k}\right|_{q}=[m]\left|P_{m-1, k-1}\right|_{q}$ for $k>1$. Denote by $P_{m, k}^{(r)}$ the subset of $P_{m, k}$ where the first element of $A$ is placed into the $r$ th element of $B$. It is easy to see that $\left|P_{m, k}^{(r)}\right|_{q}=q^{r-1}\left|P_{m-1, k-1}\right|_{q}$. Equation 17 follows because $P_{m, k}=\biguplus_{r=1}^{m} P_{m, k}^{(r)}$. Notice that every element $p \in P_{m, k}$ can be identified with a pair $\left(\hat{p}, p^{\prime}\right)$, where $\hat{p} \in \widetilde{P}_{m, k}$ and $p^{\prime}$ is a placement in $P_{k, k}$. Since $w(p)=w(\hat{p}) w\left(p^{\prime}\right)$, we have that $\left|\widetilde{P}_{m, k}\right|_{q}\left|P_{k, k}\right|_{q}=\left|P_{m, k}\right|_{q}$ which concludes the proof of 18 .

Definition 3 Consider a set of $n$ bugs, the first one of type $\left(b_{1}, f_{1}\right)$ and feet labelled with labels in $\left(0, f_{1}\right]$, the second one of type $\left(b_{2}, f_{2}\right)$ with labels in $\left(f_{1}, f_{1}+f_{2}\right]$, the third one of type $\left(b_{3}, f_{3}\right)$ with labels in $\left(f_{1}+f_{2}, f_{1}+f_{2}+f_{3}\right]$ and so on. A colony is one of the possible ways of organizing the bugs using the following procedure. The first bug stands on the ground. Once the $(j-1)$ th bug is placed, the $j$ th is placed by putting some of its $f_{j}$ feet on the ground and each one of the rest in one of the empty cells of the bodies of the preceding bugs. The pair of sequences $(\mathbf{b}, \mathbf{f}), \mathbf{b}=\left(b_{1}, b_{2}, \ldots, b_{n}\right), \mathbf{f}=\left(f_{1}, f_{2}, \ldots, f_{k}\right)$, is called the type of the colony. The legs of the colony standing on the ground are called free (see figure 3 ).

A settlement is a colony with no free legs. As a consequence of the definition of settlement, its first bug has to be a worm (placed on the ground). The subjacent colony of a settlement is the one obtained by deleting the worm and placing the corresponding feet on the ground in increasing order of the labels. A settlement whose first bug has exactly $m$ cells will be referred to as an $m$-settlement. The type of a settlement is defined to be the type of its subjacent colony (see figure 4 ).

In order to $q$-enumerate colonies (and settlements) in a convenient way we shall follow these conventions when drawing them:

1. The body of each new bug is put above all the bugs already placed.

2. Each new free leg is placed at the right hand side of the free legs already placed.

3. If one foot of the $j$ th bug is placed in one cell of the $i$ th bug, the arc of its leg goes to the right hand side of the bodies of the bugs $j-1, j-2, \ldots, i+1$.

4. The upper ends of the legs of each bug are placed from left to right following the order of the feet labels.

5. After having placed all the bugs, a ghost bug is placed in such a way that its legs do not cross among themselves and they occupy all the empty cells of the colony. The cells occupied by this ghost's legs are called free cells. 


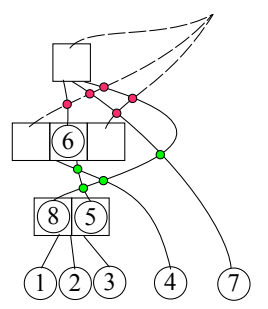

Fig. 3: Colony of type $(\mathbf{b}, \mathbf{f})=((2,3,1),(3,2,3)), 5$ free legs, and weight $q^{9}$.

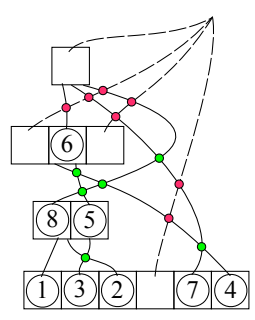

Fig. 4: A 6 -settlement of type $(\mathbf{b}, \mathbf{f})=((2,3,1),(3,2,3))$ and weight $q^{13}$.

Definition 4 The weight of a colony $C, w(C)$, is defined to be $q^{\sharp C}$, where $\sharp C$ is the total number of crossings in the colony. Observe that the weight of an $m$-settlement $S, w(S)$, is the weight of the subjacent colony times the weight of the placement of the free legs into the $m$ ground cells. Denote respectively by $\mathcal{C}_{\mathbf{b}, \mathbf{f}}(k)$ and by $\mathcal{T}_{\mathbf{b}, \mathbf{f}}(m)$ the set of colonies of type $(\mathbf{b}, \mathbf{f})$ with exactly $k$ free legs and the set of $m$ settlements of type $(\mathbf{b}, \mathbf{f})$. The q-cardinal of each of them is defined as the sum of the weights of their respective elements,

$$
\begin{aligned}
\left|\mathcal{C}_{\mathbf{b}, \mathbf{f}}(k)\right|_{q} & =\sum_{C \in \mathcal{C}_{\mathbf{b}, \mathbf{f}}(k)} w(C) \\
\left|\mathcal{T}_{\mathbf{b}, \mathbf{f}}(m)\right|_{q} & =\sum_{S \in \mathcal{T}_{\mathbf{b}, \mathbf{f}}(m)} w(S)
\end{aligned}
$$

The following is the main result of this section. Its proof is omitted in this abstract due to restrictions on its size.

Theorem 1 The q-cardinal of the set of colonies of type $(\mathbf{b}, \mathbf{f})=\left(\left(b_{1}, \ldots, b_{n}\right),\left(f_{1}, \ldots, f_{n}\right)\right)$ and $k$ free legs is equal to the q-generalized Stirling number $S_{\mathbf{b}, \mathbf{f}}^{q}(k)$, and the $q$-cardinal of the set of $m$-settlements of type $(\mathbf{b}, \mathbf{f})$ is equal to

$$
p_{\mathbf{b}, \mathbf{f}}^{q}(m)=\prod_{j=1}^{n}\left[m+d_{j-1}\right]_{f_{j}}
$$




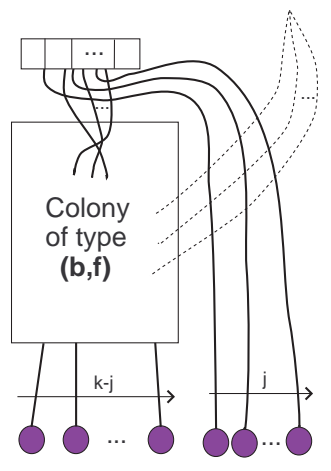

Fig. 5: Schematic drawing representing a colony of type $\left(\mathbf{b} \uplus b_{n+1}, \mathbf{f} \uplus f_{n+1}\right)$.

where $d_{j}=\sum_{i=1}^{j}\left(b_{i}-f_{i}\right)$ and $d_{0}=0$. In other words, we have the following identities:

$$
\begin{aligned}
\left|\mathcal{C}_{\mathbf{b}, \mathbf{f}}(k)\right|_{q} & =S_{\mathbf{b}, \mathbf{f}}^{q}(k) \\
\left|\mathcal{T}_{\mathbf{b}, \mathbf{f}}(m)\right|_{q} & =p_{\mathbf{b}, \mathbf{f}}^{q}(m)
\end{aligned}
$$

Theorem 2 Let $\mathbf{b}=\left(b_{1}, \ldots, b_{n}\right)$ and $\mathbf{f}=\left(f_{1}, \ldots, f_{n}\right)$ be finite sequences of non-negative integers, and set $d_{n}=\sum_{i=1}^{n}\left(b_{i}-f_{i}\right)$. We introduce the notation $\mathbf{b} \uplus b_{n+1}=\left(b_{1}, b_{2}, \ldots, b_{n}, b_{n+1}\right)$ and $\mathbf{f} \uplus f_{n+1}=$ $\left(f_{1}, f_{2}, \ldots, f_{n}, f_{n+1}\right)$. The q-generalized Stirling numbers satisfy the following recursive formula

$$
S_{\mathbf{b} \uplus b_{n+1}, \mathbf{f} \uplus f_{n+1}}^{q}(k)=\sum_{j}\left[\begin{array}{c}
f_{n+1} \\
j
\end{array}\right]\left[d_{n}+k-j\right]_{f_{n+1}-j} q^{j\left(d_{n}+k-f_{n+1}\right)} S_{\mathbf{b}, \mathbf{f}}^{q}(k-j)
$$

Proof: Every colony $C_{n+1}$ in $\mathcal{C}_{\mathbf{b} \uplus b_{n+1}, \mathbf{f} \uplus f_{n+1}}(k)$ is obtained by placing a bug $B_{n+1}$ of type $\left(b_{n+1}, f_{n+1}\right)$ on a colony $C_{n}$ of type $(\mathbf{b}, \mathbf{f})$. If the bug has exactly $j$ legs on the ground, $C_{n}$ has to be in $\mathcal{C}_{\mathbf{b}, \mathbf{f}}(k-j)$. The number of crossings in $C_{n+1}$ is equal to $w\left(C_{n}\right) q^{\operatorname{nc}\left(p\left(B_{n+1}\right)\right)}$, where $\operatorname{nc}\left(p\left(B_{n+1}\right)\right)$ is the number of new crossing produced by the placement $p$ of the feet of the bug $B_{n+1}$. Hence

$$
\begin{aligned}
S_{\mathbf{b} \uplus b_{n+1}, \mathbf{f} \uplus f_{n+1}}^{q}=\left|\mathcal{C}_{\mathbf{b} \uplus b_{n+1}, \mathbf{f} \uplus f_{n+1}}(k)\right|_{q} & =\sum_{j=0}^{f_{n+1}} \sum_{C_{n} \in \mathcal{C}_{\mathbf{b}, \mathbf{f}}(k-j)} \sum_{p} w\left(C_{n}\right) q^{\mathrm{nc}\left(p\left(B_{n+1}\right)\right)} \\
& =\sum_{j=0}^{f_{n+1}}\left|\mathcal{C}_{\mathbf{b}, \mathbf{f}}(k-j)\right|_{q} \sum_{p} q^{\mathrm{nc}\left(p\left(B_{n+1}\right)\right)} \\
& =\sum_{j=0}^{f_{n+1}} S_{\mathbf{b}, \mathbf{f}}^{q}(k-j) \sum_{p} q^{\operatorname{nc}\left(p\left(B_{n+1}\right)\right)},
\end{aligned}
$$

where the internal sum ranges over the set of all possible placements $p$ of $f_{n+1}-j$ feet of $B_{n+1}$ into the free cells of $C_{n}$. The number of free cells in each colony $C_{n} \in \mathcal{C}_{\mathbf{b}, \mathbf{f}}(k-j)$ is equal to $\left(\sum_{i=1}^{n} b_{i}-f_{i}\right)+$ 
$k-j=d_{n}+k-j$ (Number of cells - Number of legs + Number of free legs). The number of free cells in each colony $C_{n}$ after placing the feet of $B_{n+1}$ is equal to $d_{n}+k-j-\left(f_{n+1}-j\right)=d_{n}+k-f_{n+1}$. We claim that

$$
\begin{aligned}
\sum_{p} q^{\mathrm{nc}\left(p\left(B_{n+1}\right)\right)} & =\left|\widetilde{P}_{f_{n+1}, j}\right|_{q}\left|P_{f_{n+1}-j, d_{n}+k-j}\right|_{q} q^{j\left(d_{n}+k-f_{n+1}\right)} \\
& =\left[\begin{array}{c}
f_{n+1} \\
j
\end{array}\right]\left[d_{n}+k-j\right]_{f_{n+1}-j} q^{j\left(d_{n}+k-f_{n+1}\right)} .
\end{aligned}
$$

This is because $\left|\widetilde{P}_{f_{n+1}, j}\right|_{q} q$-counts the crossings when choosing the set of $j$ legs to be placed on the ground (a placement without internal crossings). $\left|P_{f_{n+1}-j, d_{n}+k-j}\right|_{q} q$-counts the crossings when placing the $f_{n+1}-j$ feet into the $d_{n}+k-j$ free cells of $C_{n}$. Finally, $q^{j\left(d_{n}+k-f_{n+1}\right)}$ is the number of crossings of the $j$ ground legs of $B_{n+1}$ with the ghost legs that occupy the $d_{n}+k-f_{n+1}$ free cells of $C_{n}$ after placing $B_{n+1}$ (see figure 5 .

$\square$ Using a similar argument, we obtain a more general recursive formula:

Theorem 3 Let $\mathbf{b}=\left(b_{1}, \ldots, b_{n}\right), \mathbf{f}=\left(f_{1}, \ldots, f_{n}\right), \mathbf{b}^{\prime}=\left(b_{1}^{\prime}, \ldots, b_{m}^{\prime}\right)$ and $\mathbf{f}^{\prime}=\left(f_{1}^{\prime}, \ldots, f_{m}^{\prime}\right)$ be finite sequences of non-negative integers. Set $d_{n}=\sum_{i=1}^{n}\left(b_{i}-f_{i}\right)$. We introduce the notation $\mathbf{b} \uplus \mathbf{b}^{\prime}=$ $\left(b_{1}, \ldots, b_{n}, b_{1}^{\prime}, \ldots, b_{m}^{\prime}\right)$ and $\mathbf{f} \uplus \mathbf{f}^{\prime}=\left(f_{1}, \ldots, f_{n}, f_{1}^{\prime}, \ldots, f_{m}^{\prime}\right)$. We have the following recursive relation for the q-generalized Stirling numbers

$$
S_{\mathbf{b} \uplus \mathbf{b}^{\prime}, \mathbf{f} \uplus \mathbf{f}^{\prime}}^{q}(k)=\sum_{l} \sum_{j}\left[\begin{array}{l}
l \\
j
\end{array}\right]\left[d_{n}+k-j\right]_{l-j} q^{j\left(d_{n}+k-l\right)} S_{\mathbf{b}, \mathbf{f}}^{q}(k-j) S_{\mathbf{b}^{\prime}, \mathbf{f}^{\prime}}^{q}(l)
$$

Example 1 Consider the case all the bugs are of type $(1,1),(\mathbf{b}, \mathbf{f})=((1,1, \ldots, 1),(1,1, \ldots, 1))$. The Stirling numbers $S_{\mathbf{b}, \mathbf{f}}^{q}(k)=S^{q}(n, k)$ are one of the two types of the classical $q$-Stirling numbers of the second kind studied in the literature (see [4]). We recover the recursive formulas

$$
\begin{gathered}
S^{q}(n+1, k)=[k] S^{q}(n, k)+q^{k-1} S^{q}(n, k-1) . \\
S^{q}(n+m, k)=\sum_{l=1}^{\min \{m, k\}} \sum_{j=0}^{l}\left[\begin{array}{l}
l \\
j
\end{array}\right][k-j]_{l-j} q^{j(k-l)} S^{q}(n, k-j) S^{q}(m, l)
\end{gathered}
$$

The combinatorial interpretation of $S^{q}(n, k)$ in terms of colonies of $n$ bugs of type $(1,1)$ with $k$ free legs is equivalent to the intertwining combinatorial interpretation in [2], and also to the definition by Garsia and Remmel in [3].

Example 2 In the case where all the bugs are of type $(r, 1)$, the colonies can be represented as forests of r-ary increasing trees (see [9], section $\mathrm{v})$. Using the notation $S_{(r, 1)^{n}}^{q}(k)=S_{(r, r, \ldots, r),(1,1, \ldots, 1)}^{q}(k)$, the recursive formulas give us

$$
\begin{gathered}
S_{(r, 1)^{n+1}}^{q}(k)=[n(r-1)+k] S_{(r, 1)^{n}}^{q}(k)+q^{(n(r-1)+k-1)} S_{(r, 1)^{n}}^{q}(k-1) . \\
S_{(r, 1)^{n+m}}^{q}(k)=\sum_{l} \sum_{j=0}^{l}\left[\begin{array}{l}
l \\
j
\end{array}\right][n(r-1)+k-j]_{l-j} q^{j(n(r-1)+k-l)} S_{(r, 1)^{n}}^{q}(k-j) S_{(r, 1)^{m}}^{q}(l)
\end{gathered}
$$




\section{Bijection with $q$-rook placements}

Each colony $C$ of type $(\mathbf{b}, \mathbf{f})=\left(\left(b_{1}, b_{2}, \ldots, b_{n}\right),\left(f_{1}, f_{2}, \ldots, f_{n}\right)\right)$ with exactly $k$ free legs, can be uniquely identified with a placement of $|\mathbf{f}|-k=f_{1}+\cdots+f_{n}-k$ rooks on a Ferrers board $F_{\mathbf{b}, \mathbf{f}}$. The board $F_{\mathbf{b}, \mathbf{f}}$ is outlined (using the French convention) by successively drawing $f_{j}$ unitary segments downwards and $b_{j}$ unitary segments to the right, for $j=1,2, \ldots, n$. We number the horizontal segments in the order we draw them, as well as the vertical segments. We then assign to each row of the board, the number of its corresponding vertical segment, and to each column, the number of its corresponding horizontal segment. Flat sections of the board corresponding to $f_{1}$ and $b_{n}$ can be ignored. Assuming that the cells of the bugs of the colony are numbered from right to left and from bottom to top, we place a rook on the cell $(r, c)$ (row $r$, column $c$ ) of $F_{\mathbf{b}, \mathbf{f}}$ if and only if the foot $r$ is in the cell $c$ in $C$. This forms a $(|\mathbf{f}|-k)$-rook placement on $F_{\mathbf{b}, \mathbf{f}}$. This relation is well defined and bijective.

Theorem 4 The bijection defined above preserves the q-weight of the structures, defined for rook placements as $q^{l}$ where $l$ is the number of empty cells in the Ferrers board that do not have a rook above or to their right (See [13] and [3]). Notice that the convention used here to draw the Ferrers board and define the q-weight of rook placements is the French one. Other authors might prefer other coventions.

This bijection gives a combinatorial interpretation of the equality

$$
R_{f_{1}+f_{2}+\cdots+f_{n}-k}\left(F_{\mathbf{b}, \mathbf{f}}, q\right)=S_{\mathbf{b}, \mathbf{f}}^{q}(k)
$$

where $R_{m}(B, q)$ is defined as in [13].

\section{Dual graded graphs}

In this section we relate our work to that of Fomin [14]. For this we shall introduce some notation

Definition $5 A$ graded graph is a triple $G=(P, \rho, E)$ where $P$ is a discrete set of vertices, $\rho: P \rightarrow \mathbb{Z}$ is a rank function, and $E$ is a multiset of edges $(x, y)$ where $\rho(y)=\rho(x)+1$. The set $P_{n}=\{x: \rho(x)=n\}$ is called a level of $G$.

As in Fomin's work, levels are assumed to be finite. Some levels could be empty. In case

$$
P_{0}=\{\hat{0}\}, \quad P_{-1}=P_{-2}=\cdots=\emptyset
$$

we say $G$ is a graph with a zero $\hat{0}$.

For $x, y \in P$, let $e(x \rightarrow y)$ denote the number of shortest (passing through at most one vertex in each level) non-oriented paths between $x$ and $y$. In a graph with a zero, denote $e(y)=e(\hat{0} \rightarrow y)$. Define

$$
\alpha(n \rightarrow m)=\sum_{x \in P_{n}} \sum_{y \in P_{m}} e(x \rightarrow y)
$$

This is, the number of paths connecting the $n$th and $m$ th levels. One could similarly define $\alpha(n \rightarrow$ $m \rightarrow l), e(x \rightarrow y \rightarrow z)$, etc.

In his work, Fomin obtains remarkable combinatorial results regarding path counting in graded graphs, through the use of two operators called up and down operators that act over the freely generated vector 
space $K P$ ( $K$ being a field). In the main special case of his work, these operators satisfy commutation relations very similar to the one satisfied by the operators $D_{q}$ and $X$.

Let $G=(P, \rho, E)$ be a graded graph and $K$ be a field of characteristic zero. The formal linear combinations of vertices in $P$ with coefficients in $K$ form the vector space $K P$. For any $x, y \in P$, let $a(x, y)$ denote the multiplicity of $(x, y)$, i.e., the number of edges joining $x$ and $y$. The linear operators $\mathcal{U}$ and $\mathcal{U}^{*}$ are defined by

$$
\mathcal{U} x=\sum_{\rho(y)=\rho(x)+1} a(x, y) y
$$

and

$$
\left(\mathcal{U}^{*}\right) y=\sum_{\rho(x)=\rho(y)-1} a(x, y) x
$$

Definition 6 Let $G_{1}=\left(P, \rho, E_{1}\right)$ and $G_{2}=\left(P, \rho, E_{2}\right)$ be a pair of graded graphs with a common set of vertices and a common rank function. Define an oriented graded graph $G=\left(P, \rho, E_{1}, E_{2}\right)$ by directing the $G_{1}$-edges up (in the direction of increasing rank) and the $G_{2}$-edges down (in the direction of decreasing rank). The up and down operators $\mathcal{U}, \mathcal{D} \in \operatorname{End}(K P)$ are defined by

$$
\mathcal{U} x=\sum_{\rho(y)=\rho(x)+1} a_{1}(x, y) y
$$

and

$$
\mathcal{D} y=\sum_{\rho(x)=\rho(y)-1} a_{2}(x, y) x
$$

where $a_{i}(x, y)$ is the multiplicity of the edge $(x, y)$ in $G_{i}$.

Definition 7 Let $G$ be as in definition 6 A path in $G$ is an oriented path that respects the orientations of the arcs. Assume $\mathcal{W}$ is a word in the alphabet $\{\mathcal{U}, \mathcal{D}\}$. A path in $G$ is a $\mathcal{W}$-path if its consecutive arcs are directed up or down in accordance with the word $\mathcal{W}$. The $\mathcal{U}_{s}$ correspond to the up-directed arcs of $G_{1}$ and the $\mathcal{D}$ s to the down-directed arcs of $G_{2}$. The word $\mathcal{W}$ is to be read from right to left. We denote by $e_{\mathcal{W}}(x \rightarrow y)$ and $e_{\mathcal{W}}(y)$ the number of $\mathcal{W}$-paths from $x$ to $y$ and the number of $\mathcal{W}$-paths from $\hat{0}$ to $y$ (in case $G$ is a graph with zero), respectively. The numbers $\alpha_{\mathcal{W}}(m \rightarrow n)$ are defined in a similar way.

The following proposition is immediate from the two previous definitions.

Proposition 3 The number of $\mathcal{W}$-paths in $G$ from $x$ to $y$ is the coefficient of $y$ in the expansion of $\mathcal{W} x$. This is,

$$
\mathcal{W} x=\sum_{y \in P} e_{\mathcal{W}}(x \rightarrow y) y
$$

We will focus on the following situation: 
Definition 8 Let $G_{1}$ and $G_{2}$ be graded graphs as in definition 6. Let $q, r$ be elements of the field K. $G_{1}$ and $G_{2}$ are said to be $q, r$-dual if they satisfy

$$
\mathcal{D U}=q \mathcal{U D}+r I
$$

where $I$ is the identity operator over $K P$. In the case $q, r=1$ we say $G_{1}$ and $G_{2}$ are simply dual. $G_{1}$ is said to be self- $q, r$-dual (respectively self-dual) if it is $q, r$-dual (respectively dual) to itself.

The commutation rule of equation (34) is the same satisfied by the operators $r X$ and $D_{q}$. As a consequence,

$$
\mathcal{W}_{\mathbf{b}, \mathbf{f}}=\mathcal{U}^{b_{n}} \mathcal{D}^{f_{n}} \cdots \mathcal{U}^{b_{1}} \mathcal{D}^{f_{1}}=\sum_{k} r^{|\mathbf{f}|-k} S_{\mathbf{b}, \mathbf{f}}^{q}(k) \mathcal{U}^{d_{n}+k} \mathcal{D}^{k} .
$$

Applying this operator to $x \in P$ and taking the coefficient of $y \in P$ on both sides of the equality, we obtain the following theorem,

Theorem 5 Let $G=\left(P, \rho, E_{1}, E_{2}\right)$ be a graph as in definition 6 such that $G_{1}$ and $G_{2}$ are $q$, $r$-dual. Set $\mathbf{b}=\left(b_{1}, \ldots, b_{n}\right), \mathbf{f}=\left(f_{1}, \ldots, f_{n}\right), d_{n}=|\mathbf{b}|-|\mathbf{f}|=\sum_{i=1}^{n}\left(b_{i}-f_{i}\right)$, and $\mathcal{W}=\mathcal{U}^{b_{n}} \mathcal{D}^{f_{n}} \ldots \mathcal{U}^{b_{1}} \mathcal{D}^{f_{1}}$. Then,

$$
e_{\mathcal{W}}(x \rightarrow y)=\sum_{k} r^{|\mathbf{f}|-k} S_{\mathbf{b}, \mathbf{f}}^{q}(k) e_{\mathcal{U}^{d_{n}+k}} \mathcal{D}^{k}(x \rightarrow y)
$$

and

$$
\alpha_{\mathcal{W}}\left(l \rightarrow l+d_{n}\right)=\sum_{k} r^{|\mathbf{f}|-k} S_{\mathbf{b}, \mathbf{f}}^{q}(k) \alpha_{\mathcal{U}^{d_{n}+k} \mathcal{D}^{k}}\left(l \rightarrow l+d_{n}\right)
$$

for any integer $l$.

This result gives a formula for counting complicated paths in terms of $\operatorname{simpler} \mathcal{U}^{d_{n}+k} \mathcal{D}^{k}$-paths. In the case $G$ is a graph with zero we can set $x=\hat{0}$ and use $\mathcal{W}=\mathcal{U}^{b_{n}} \mathcal{D}^{f_{n}} \ldots \mathcal{U}^{b_{1}} \mathcal{D}^{f_{1}} \mathcal{U}^{m}$ for some $m>0$ (otherwise there would be no $\mathcal{W}$-paths starting in $\hat{0}$ ), obtaining,

$$
e_{\mathcal{W}}(y)=\sum_{k} r^{|\mathbf{f}|-k} S_{m \uplus \mathbf{b}, 0 \uplus \mathbf{f}}^{q}(k) e_{\mathcal{U}^{d_{n}+m+k}} \mathcal{D}^{k}(y)
$$

There are no $\mathcal{D}$-paths starting in $\hat{0}$, so the only non-zero term in the sum of equation 38 is the one corresponding to $k=0$. Also $S_{m \uplus \mathbf{b}, 0 \uplus \mathbf{f}}^{q}(0)=p_{\mathbf{b}, \mathbf{f}}^{q}(m)$ as a direct result of the combinatorial interpretations of $S_{\mathbf{b}, \mathbf{f}}^{q}(k)$ and $p_{\mathbf{b}, \mathbf{f}}^{q}(m)$ outlined in section 3. These observations yield the following result:

Corollary 3 Let $G=\left(P, \rho, E_{1}, E_{2}\right)$ be a graph as in definition 6 such that $G_{1}$ and $G_{2}$ are q,r-dual. Assume $G$ has a zero. Set $\mathbf{b}=\left(b_{1}, \ldots, b_{n}\right), \mathbf{f}=\left(f_{1}, \ldots, f_{n}\right), d_{j}=\sum_{i=1}^{j}\left(b_{j}-f_{j}\right), d_{0}=0,|\mathbf{f}|=$ $f_{1}+\cdots+f_{n}$, and $\mathcal{W}=\mathcal{U}^{b_{n}} \mathcal{D}^{f_{n}} \cdots \mathcal{U}^{b_{1}} \mathcal{D}^{f_{1}} \mathcal{U}^{m}$ for some $m>0$. Assume $d_{n}+m>0$ and let $y$ be any vertex of rank $d_{n}+m$ in $P$. Then,

$$
e_{\mathcal{W}}(y)=r^{|\mathbf{f}|} p_{\mathbf{b}, \mathbf{f}}^{q}(m) e(y)
$$

and

$$
\alpha_{\mathcal{W}}\left(0 \rightarrow d_{n}+m\right)=r^{|\mathbf{f}|} p_{\mathbf{b}, \mathbf{f}}^{q}(m) \alpha\left(0 \rightarrow d_{n}+m\right)
$$

where $p_{\mathbf{b}, \mathbf{f}}^{q}(m)=\prod_{j=1}^{n}\left[m+d_{j-1}\right]_{f_{j}}$. 


\section{$6 p, q$-Analogs}

Recall the definition of the $p, q$-analog of the derivative operator, $D_{p, q}$;

$$
D_{p, q} f(x)=\frac{f(p x)-f(q x)}{(p-q) x}
$$

$X$ and $D_{p, q}$ satisfy the commutation rule

$$
D_{p, q} X=q X D_{p, q}+T_{p}
$$

where $T_{p}$ is defined by $T_{p} f(x)=f(p x)$. We define the $p, q$-generalized Sitrling numbers as the coefficients $S_{\mathbf{b}, \mathbf{f}}^{p, q}(k)$ (rational functions in $p$ and $q$ ) appearing in the straightening formula

$$
w_{\mathbf{b}, \mathbf{f}}^{p, q}=X^{b_{n}} D_{p, q}^{f_{n}} \cdots X^{b_{1}} D_{p, q}^{f_{1}}=\sum_{k} S_{\mathbf{b}, \mathbf{f}}^{p, q}(k) T_{p}^{l_{k}} X^{d_{n}+k} D_{p, q}^{k}
$$

where the $l_{k}$ are integers. We easily verify the following relations:

$$
\begin{gathered}
T_{p} X=p X T_{p} \\
D_{r} T_{p}=p T_{p} D_{r} \\
D_{p, q}=T_{p} D_{q / p} \text { and more generally } D_{p, q}^{f}=T_{p}^{\left(\begin{array}{c}
f \\
2
\end{array}\right)} D_{q / p}^{f} \text { for } f \in \mathbb{Z}^{+}
\end{gathered}
$$

which yield:

$$
w_{\mathbf{b}, \mathbf{f}}^{p, q}=p^{m} T_{p}^{l} X^{b_{n}} D_{q / p}^{f_{n}} \cdots X^{b_{1}} D_{q / p}^{f_{1}}
$$

where $m=\sum_{j=1}^{n}\left(d_{j}-d_{n}-b_{j}\right)\left(\begin{array}{c}f_{j} \\ 2\end{array}\right)$ and $l=\sum_{j=1}^{n}\left(\begin{array}{c}f_{j} \\ 2\end{array}\right)$. Thus,

$$
\begin{aligned}
w_{\mathbf{b}, \mathbf{f}}^{p, q} & =\sum_{k} p^{m} S_{\mathbf{b}, \mathbf{f}}^{q / p}(k) T_{p}^{l} X^{d_{n}+k} D_{q / p}^{k} \\
& =\sum_{k} p^{m+\left(d_{n}+k\right)\left(\begin{array}{l}
k \\
2
\end{array}\right)} S_{\mathbf{b}, \mathbf{f}}^{q / p}(k) T_{p}^{l-\left(\begin{array}{c}
k \\
2
\end{array}\right)} X^{d_{n}+k} T_{p}^{\left(\begin{array}{c}
k \\
2
\end{array}\right)} D_{q / p}^{k} \\
& =\sum_{k} p^{m+\left(d_{n}+k\right)\left(\begin{array}{l}
k \\
2
\end{array}\right)} S_{\mathbf{b}, \mathbf{f}}^{q / p}(k) T_{p}^{l-\left(\begin{array}{c}
k \\
2
\end{array}\right)} X^{d_{n}+k} D_{p, q}^{k}
\end{aligned}
$$

This is,

$$
S_{\mathbf{b}, \mathbf{f}}^{p, q}(k)=p^{m+\left(d_{n}+k\right)\left(\begin{array}{l}
k \\
2
\end{array}\right)} S_{\mathbf{b}, \mathbf{f}}^{q / p}(k)
$$

\section{Acknowledgements}

The authors would like to thank the referees for their useful suggestions on the presentation of this material, and François Bergeron for pointing out essential elements towards the development of this work. 


\section{References}

[1] P. Blasiak, A. Horzela, K. Penson, G. Duchamp and A. Solomon, Boson Normal Ordering via Substitutions and Sheffer-type Polynomials, Phys. Lett. A (2005)

[2] R. Ehrenborg and M. Readdy, Juggling and applications to q-analogues, Discrete Math. 157 (1996), 107-125.

[3] A. Garsia and J. Remmel, $q$-Counting rook configuration and a formula of Frobenius, J. Combin. Theory Ser. A 41 (1986), 246-275.

[4] H. Gould, The $q$-Stirling numbers of the first and second kinds, Duke Math. J. 32, (1961), 281-289.

[5] J. Katriel, Bell numbers and coherent states, Phys. Lett. A 237 (2000), 159-161.

[6] Katriel J. and Kibler M., Normal ordering for deformed boson operators and operator-valued deformed Stirling numbers, J. Phys. A: Math. Gen. 25 (1992), 2683-2691.

[7] J. Katriel and G. Duchamp, Ordering relations for $q$-boson oprators, continued fractions techniques, and the $q$-CBH enigma, J. of Phys. A 28 7209-7225 (1995).

[8] T. Mansour, M. Shork and S. Severini, Wicks's theorem for $q$-deformed boson operators, Preprint arXiv:quant-ph/0703086v1 (2007).

[9] M. Méndez, P. Blasiak, K. Penson, Combinatorial approach to generalized Bell and Stirling numbers and boson normal ordering problem, J. Math. Phys., 46, 1 (2005).

[10] A. M. Navon, Combinatorics and fermion algebra, Nuovo Cimento 16 (1973), 324-330.

[11] M. Schork, Normal ordering q-bosons and combinatorics, Phys. Lett. A 355 (2006), 293-297.

[12] M. Schork, On the combinatorics of normal ordering bosonic operators and deformations of it, $J$. Phys. A: Math. Gen. 36 (2003) 4651-4665.

[13] A. Varvak, Rook numbers and the normal ordering problem, in 16th Annual International Conference on Formal Power Series and Algebraic Combinatorics (Vancouver B.C., Canada) (2004), arXiv:math.CO/0402376

[14] S. Fomin, Duality of Graded Graphs, J. Alg. Combin., 3 (1994), 357-404. 
